\title{
Debye Series Analysis of Scattering of a Plane Wave by a Spherical Bragg Grating
}

James A. Lock

Cleveland State University, j.lock@csuohio.edu

Follow this and additional works at: https://engagedscholarship.csuohio.edu/sciphysics_facpub

Part of the Physics Commons

How does access to this work benefit you? Let us know!

\section{Publisher's Statement}

This paper was published in Applied Optics and is made available as an electronic reprint with the permission of OSA. The paper can be found at the following URL on the OSA website: http://www.opticsinfobase.org/ao/abstract.cfm?URI=ao-44-26-5594. Systematic or multiple reproduction or distribution to multiple locations via electronic or other means is prohibited and is subject to penalties under law.

\section{Original Citation}

Lock, James A. "Debye Series Analysis of Scattering of a Plane Wave by a Spherical Bragg Grating." Applied Optics 44 (2005): 5594-5603.

\section{Repository Citation}

Lock, James A., "Debye Series Analysis of Scattering of a Plane Wave by a Spherical Bragg Grating" (2005). Physics Faculty Publications. 115.

https://engagedscholarship.csuohio.edu/sciphysics_facpub/115

This Article is brought to you for free and open access by the Physics Department at EngagedScholarship@CSU. It has been accepted for inclusion in Physics Faculty Publications by an authorized administrator of EngagedScholarship@CSU. For more information, please contact library.es@csuohio.edu. 


\title{
Debye series analysis of scattering of a plane wave by a spherical Bragg grating
}

\author{
James A. Lock
}

\begin{abstract}
The Debye series decomposition of the partial-wave scattering amplitudes of a multilayer sphere is derived. The partial-wave transmission and reflection terms appearing in the Debye series are multiplescattering amplitudes written in terms of four basic quantities and combined together layer by layer in an identical way. The resulting expressions are then used to calculate the scattered intensity of a spherical Bragg grating covering a dielectric core particle and to analyze a number of new structures appearing in the scattered intensity. (C) 2005 Optical Society of America
\end{abstract}

OCIS codes: $230.1480,230.4170,290.4020$.

\section{Introduction}

In many situations, ranging from climate modeling ${ }^{1}$ to combustion studies, ${ }^{2}$ there is great interest in the electromagnetic scattering properties of radially inhomogeneous spheres. These investigations have most often been handled by decomposing the sphere into $M$ homogeneous concentric layers with the refractive index being constant in each layer. The collection of layers forms a histogram approximation to the radial inhomogeneity. The first successful attack on this problem in the context of Mie theory was Aden and Kerker's solution to scattering by a coated sphere, ${ }^{3,4}$ i.e., $M=2$. Bhandari's solution for scattering by an $M$-layer sphere ${ }^{5}$ proceeded by analogy to the calculation of transmission and reflection of a plane wave by a stack of flat slabs. ${ }^{6,7}$ For the flat slab problem the transmission and reflection coefficients are obtained iteratively, beginning with the first slab, adding the second slab, the third, and so on, all the way to the last slab. Similarly, in the $M$-layer sphere problem various partial-wave amplitudes are iterated from the core of the sphere to its outer surface, adding on another layer at each iteration. If the number of layers are large or if the refractive indices have a large imaginary part, 8 this progressive iteration procedure is prone to numerical overflow and under-

James Lock (j.lock@csuohio.edu) is with the Department of Physics, Cleveland State University, 2121 Euclid Avenue, Cleveland, Ohio 44115-2440.

Received 21 January 2005; revised manuscript received 15 April 2005; accepted 29 March 2005.

0003-6935/05/265594-10\$15.00/0

(C) 2005 Optical Society of America flow problems. As a result, much effort has gone into constructing numerically stable algorithms for computing scattering, absorption, and the internal fields of a multilayer sphere. ${ }^{9-13}$ In the context of multiple scattering, the multilayer sphere partial-wave scattering amplitudes have been written in terms of homogeneous sphere scattering amplitudes, ${ }^{14-16}$ producing an order of scattering series. The properties of morphology-dependent resonances (MDRs) in multilayer spheres have also been examined. ${ }^{1}$

For scattering by a large homogeneous sphere, the many participating partial waves begin to organize themselves into raylike behaviors. The connection between wave scattering and ray scattering in the large sphere or short-wavelength limit is made through the Debye series expansion in which each partial-wave scattering amplitude is written as a sum of diffraction of the partial wave, its external reflection from the sphere surface, and transmission through the sphere following all numbers of internal reflections from the surface. The Debye series was initially formulated for scattering of a normally incident plane wave by a cylinder ${ }^{17}$ and has been subsequently extended to scattering by a sphere, ${ }^{18-20}$ the internal fields, ${ }^{21}$ scattering by a coated sphere, ${ }^{22}$ and scattering of a plane wave diagonally incident on a cylinder. ${ }^{23}$ Perhaps the most important use of the Debye series has been to obtain a physical understanding of the various wave-scattering processes that interleaf to form the scattered intensity. If one computes and plots the Mie intensity as a function of scattering angle, the resulting graph contains a large number of oscillations of various sizes and amplitudes, each of which has a physical cause. The Debye series is usually able to ascribe a physical meaning to each oscil- 
lation in terms of diffraction, external reflection, or transmission following some given number of internal reflections if that single process dominates all the others at that scattering angle or if it is caused by the interference between two such fundamental processes whose strengths are roughly comparable at that scattering angle. Exceptions do occur, such as MDRs, which require the constructive interference of hundreds or thousands of Debye series terms to build up the resonant amplitude. But, in general, the Debye series does a thorough job in identifying the physical mechanism responsible for almost all the structure of the Mie intensity for spheres whose circumference is larger than about 50 wavelengths of the incident light. ${ }^{24}$

In order to understand the novel structures appearing in the scattered intensity of an $M$-layer sphere, in this paper I derive the Debye series decomposition of the multilayer sphere partial-wave scattering amplitudes. In Section 2, I define four basic amplitudes for each partial wave in terms of which the $M=1$ homogeneous sphere partial-wave scattering amplitudes are conveniently written. These four basic amplitudes have the virtue that the partialwave scattering amplitudes for both the coated sphere and the multilayer sphere are written in terms of their simple combinations, the combinations being identical for all $M$. These combinations physically correspond to combining two or more adjacent interfaces into a single effective scattering unit. In Section 3, I quickly review the Debye series decomposition of the homogeneous sphere partial-wave scattering amplitudes and show how the transmission and reflection amplitudes appearing in the Debye series are written in terms of the four basic amplitudes defined in Subsection 2.B. I then find that for a layered sphere with $M \geq 2$, if one groups all the interfaces together into a single effective scattering unit by combining the four basic amplitudes as described in Subsection 2.C, the transmission and reflection amplitudes between the core and the external medium have the same structural form as do the corresponding amplitudes for the $M=1$ homogeneous sphere. The form of the Debye series is structurally the same as well. In Section 4, I write the order of scattering decomposition of the coated sphere partial-wave scattering amplitudes in terms of the four basic amplitudes of Subsection 2.B. Last, in Section 5, I test these Debye series formulas on the case of a spherical core particle coated with a number of quarter-wavelength thick layers of alternately slightly higher and lower refractive index, corresponding to a spherical Bragg grating ${ }^{25,26}$ covering the core particle. The scattered intensity as a function of both scattering angle and wavelength of the incident light exhibits a number of very noticeable differences from that of a homogeneous sphere of the same outer radius. Calculation of the various terms of the Debye series for the multilayer sphere provides a solid physical understanding of these differences. This understanding can then hopefully be used as the basis for engineering composite particles with strong scattering lobes in preferential directions. ${ }^{16}$

\section{Scattering of a Plane Wave by a Multilayer Sphere}

\section{A. Geometry}

The formulas for electromagnetic scattering of a plane wave by a multilayer sphere are cumbersome because of the extensive and elaborate superscripts and subscripts. In this section I carefully explain my notation and clearly state what portion of the notation is suppressed in the formulas for the sake of convenience. Consider a sphere consisting of $M$ concentric homogeneous layers. The innermost region of the composite sphere, the core, is labeled region 1 and has refractive index $n_{1}$ and radius $A_{1}$. The layers progressively outward from the core have refractive index $n_{j}$ and radius $A_{j}$ where $2 \leq j \leq M$, with the outer radius of the multilayer sphere being $A_{M}$. Exterior to the sphere is a homogeneous medium labeled region $M+1$ having refractive index $n_{M+1}$. The center of the layered sphere is chosen to be at the origin of the coordinates. Incident on the multilayer sphere is an electromagnetic plane wave of unit amplitude, freespace wavelength $\lambda$, and wave number $k=2 \pi / \lambda$, propagating in the $+z$ direction, and with its electric field polarized in the $x$ direction. The time dependence of the plane wave is taken to be $\exp (-i \omega t)$.

\section{B. Scattering by a Homogeneous Sphere}

If $M=1$, a homogeneous sphere of refractive index $n_{1}$ and radius $A_{1}$ is in the external medium of refractive index $n_{2}$. The solution to the Mie scattering problem may be conveniently written in terms of four basic partial-wave amplitudes $N_{12, l}, D_{12, l}, P_{12, l}$, and $Q_{12, l}$, which will turn out to be convenient for describing scattering by the $M$-layer sphere as well. For transverse electric (TE) partial waves, the basic amplitudes are

$$
\begin{aligned}
& N_{12, l}=n_{1} \psi_{l}\left(X_{12}\right) \psi_{l}{ }^{\prime}\left(Y_{12}\right)-n_{2} \psi_{l}{ }^{\prime}\left(X_{12}\right) \psi_{l}\left(Y_{12}\right), \\
& D_{12, l}=n_{1} \chi_{l}\left(X_{12}\right) \psi_{l}{ }^{\prime}\left(Y_{12}\right)-n_{2} \chi_{l}{ }^{\prime}\left(X_{12}\right) \psi_{l}\left(Y_{12}\right), \\
& P_{12, l}=n_{1} \psi_{l}\left(X_{12}\right) \chi_{l}{ }^{\prime}\left(Y_{12}\right)-n_{2} \psi_{l}{ }^{\prime}\left(X_{12}\right) \chi_{l}\left(Y_{12}\right), \\
& Q_{12, l}=n_{1} \chi_{l}\left(X_{12}\right) \chi_{l}{ }^{\prime}\left(Y_{12}\right)-n_{2} \chi_{l}{ }^{\prime}\left(X_{12}\right) \chi_{l}\left(Y_{12}\right),
\end{aligned}
$$

where $\psi_{l}(w)$ is the Riccati-Bessel function ${ }^{27}$

$$
\psi_{l}(w)=w j_{l}(w)
$$

with $j_{l}(w)$ being a spherical Bessel function; $\chi_{l}(w)$ is the Riccati-Neumann function

$$
\chi_{l}(w)=w n_{l}(w)
$$

with $n_{l}(w)$ being a spherical Neumann function; the prime denotes the derivative of a function with respect to its argument, and 


$$
\begin{aligned}
& X_{12}=2 \pi n_{2} A_{1} / \lambda, \\
& Y_{12}=2 \pi n_{1} A_{1} / \lambda .
\end{aligned}
$$

For use in Section 3, the Riccati-Hankel functions $\xi_{l}^{(1)}(w)$ and $\xi_{l}^{(2)}(w)$ are

$$
\begin{aligned}
& \xi_{l}^{(1)}(w)=w h_{l}^{(1)}(w), \\
& \xi_{l}^{(2)}(w)=w h_{l}^{(2)}(w),
\end{aligned}
$$

where $h_{l}^{(1)}(w)$ and $h_{l}^{(2)}(w)$ are spherical Hankel functions. Since the time dependence is $\exp (-i \omega t)$, $\xi_{l}^{(1)}(w)$ describes an outgoing spherical wave and $\xi_{l}^{(2)}(w)$ describes an incoming spherical wave. The subscripts 1 and 2 in Eqs. (1a)-(1d), (4a), and (4b) indicate that the quantities are evaluated at the interface between regions 1 and 2 . The four basic amplitudes of Eqs. (1a)-(1d) satisfy the identity

$$
N_{12, l} Q_{12, l}-D_{12, l} P_{12, l}=n_{1} n_{2},
$$

which is a consequence of the Wronskian relation ${ }^{27}$ for the Riccati-Bessel and Riccati-Neumann functions. The four basic partial-wave amplitudes analogous to Eqs. (1a)-(1d) for transverse magnetic (TM) waves have $n_{2}$ multiplying the first product of Riccati-Bessel functions and $n_{1}$ multiplying the second product.

In the Mie scattering problem, the scalar radiation potentials inside and outside the sphere for the TE polarization are taken to be

$$
\begin{aligned}
\Psi_{1, l}(r, \theta, \phi)= & \sum_{l=1}^{\infty} n_{1}\left\{\left(i^{l}\right)(2 l+1) /[l(l+1)]\right\} d_{12, l} \\
& \times \psi_{l}\left(n_{1} k r\right) P_{l}^{1}[\cos (\theta)] \sin (\phi), \\
\Psi_{2, l}(r, \theta, \phi)= & \sum_{l=1}^{\infty} n_{2}\left\{\left(i^{l}\right)(2 l+1) /[l(l+1)]\right\}\left[\psi_{l}\left(n_{2} k r\right)\right. \\
& \left.-b_{12, l} \xi_{l}^{(1)}\left(n_{2} k r\right)\right] P_{l}^{1}[\cos (\theta)] \sin (\phi),
\end{aligned}
$$

respectively. The partial-wave scattering amplitude is $b_{12, l}$, and the partial-wave interior amplitude is $d_{12, l}$. The scalar radiation potentials for TM polarized waves are identical to Eqs. (7a) and (7b) except that the partial-wave scattering amplitude is $a_{12, l}$, the interior partial-wave amplitude is $c_{12, l}$, and the factors of $\sin (\phi)$ are replaced by $\cos (\phi)$. By matching the boundary conditions of the electric and magnetic fields at the surface of the sphere, one obtains

$$
\begin{aligned}
& a_{12, l}, b_{12, l}=N_{12, l} /\left(N_{12, l}+i D_{12, l}\right), \\
& c_{12, l}, d_{12, l}=-i n_{2} /\left(N_{12, l}+i D_{12, l}\right) .
\end{aligned}
$$

The formulas for the TE, TM, and unpolarized scattered intensity in terms of these partial-wave scattering amplitudes are given in Refs. 28-30. For the balance of this paper, I retain the partial-wave number $l$ when describing scattering by a homogeneous sphere but suppress it when describing scattering by a multilayer sphere.

\section{Scattering by a Coated Sphere}

For the $M=2$ coated sphere the four basic partialwave amplitudes of Eqs. (1a)-(1d) are combined as

$$
\begin{aligned}
& N_{123}=D_{12} N_{23}-N_{12} P_{23}, \\
& D_{123}=D_{12} D_{23}-N_{12} Q_{23}, \\
& P_{123}=Q_{12} N_{23}-P_{12} P_{23}, \\
& Q_{123}=Q_{12} D_{23}-P_{12} Q_{23},
\end{aligned}
$$

These four composite partial-wave amplitudes satisfy the identity

$$
N_{123} Q_{123}-D_{123} P_{123}=n_{1} n_{2}^{2} n_{3},
$$

which may be derived by using Eq. (6). The partialwave scattering amplitudes for the coated sphere are given in Refs. 31 and 32, and after much algebra can be brought to the form

$$
a_{123}, b_{123}=N_{123} /\left(N_{123}+i D_{123}\right) .
$$

The partial-wave amplitudes in the coating and core can also be written in terms of $N_{12}, D_{12}, P_{12}, Q_{12}, N_{23}$, $D_{23}, P_{23}$, and $Q_{23}$ once $a_{123}$ and $b_{123}$ have been determined.

\section{Scattering by a Multilayer Sphere}

The pattern for the partial-wave amplitudes seen for $M=2$ is found after much algebra to generalize to arbitrary $M$. In analogy to Eqs. (9a)-(9d), let

$$
\begin{aligned}
& N_{12 \ldots J+1}=D_{12 \ldots J} N_{J, J+1}-N_{12 \ldots J} P_{J, J+1}, \\
& D_{12 \ldots J+1}=D_{12 \ldots J} D_{J, J+1}-N_{12 \ldots J} Q_{J, J+1}, \\
& P_{12 \ldots J+1}=Q_{12 \ldots J} N_{J, J+1}-P_{12 \ldots J} P_{J, J+1}, \\
& Q_{12 \ldots J+1}=Q_{12 \ldots J} D_{J, J+1}-P_{12 \ldots J} Q_{J, J+1},
\end{aligned}
$$

with $2 \leq J \leq M$. These amplitudes are formed by combining the composite amplitudes previously obtained for the first $J-1$ interfaces with those for the $J$ interface. Specifically, first one uses Eqs. (9a)-(9d) to combine interfaces 12 and 23 into the single unit 123 via Eqs. (9a)-(9d). Then one uses Eqs. (12a)(12d) to combine unit 123 with interface 34 into the new unit 1234 . Then one combines the unit 1234 with the interface 45 into a new unit 12345 , and so on repeatedly until the final unit $12 \ldots M+1$ is formed. This is the same iteration procedure used by previous authors for both a stack of flat slabs 6,7 and the $M$-layer sphere scattering amplitudes..$^{5,9-13} \mathrm{I}$ call 
this the progressive iteration scheme since it progresses from the core of the sphere to its outer surface. The amplitudes of Eqs. (12a)-(12d) satisfy the identity

$$
N_{12 \ldots J+1} Q_{12 \ldots J+1}-D_{12 \ldots J+1} P_{12 \ldots J+1}=n_{1}\left(\prod_{j=2}^{J} n_{j}^{2}\right) n_{J+1} .
$$

Not surprisingly, the partial-wave scattering amplitudes of the $M$-layer sphere are again given in terms of these four composite amplitudes by

$a_{12 \ldots M+1}, b_{12 \ldots M+1}=N_{12 \ldots M+1} /\left(N_{12 \ldots M+1}+i D_{12 \ldots M+1}\right)$.

The interior amplitudes are obtained the same way in which they were for the $M=2$ case. ${ }^{12}$

A different procedure, which I call the parallel iteration scheme, is also an option when the number of layers of the sphere is $2^{p}$. As an example of this for $p=3$, interfaces 12 and 23,34 and 45,56 and 67 , and 78 and 89 can be combined in parallel into the units $123,345,567$, and 789 by using Eqs. (9a)-(9d). The first two of these units can then be combined into the new unit 12345 , and the second two combined in parallel into 56789 by using the same pattern of combination of the four amplitudes as was the case for the progressive iteration method:

$$
\begin{aligned}
& N_{12345}=D_{123} N_{345}-N_{123} P_{345}, \\
& D_{12345}=D_{123} D_{345}-N_{123} Q_{345}, \\
& P_{12345}=Q_{123} N_{345}-P_{123} P_{345}, \\
& Q_{12345}=Q_{123} D_{345}-P_{123} Q_{345} .
\end{aligned}
$$

Last, these two units can be similarly combined into the single unit $12 \ldots 9$. All the composite amplitudes in the parallel iteration method are found to be identical to those obtained by the progressive iteration method. The parallel iteration scheme has the possible numerical advantage that all the singleinterface amplitudes are iterated the same number of times, whereas in the progressive iteration scheme the single-interface amplitudes nearer the core are iterated many more times than those nearer the outer surface.

\section{Debye Series Decomposition of the Partial-Wave Scattering Amplitudes}

\section{A. Homogeneous Sphere}

In analogy to ray theory, the partial-wave scattering and interior amplitudes may be decomposed through the Debye series into fundamental interactions of the partial wave with the sphere surface, e.g., diffraction, external reflection, transmission, and transmission following any number of internal reflections. The partial-wave transmission and reflection amplitudes appearing in the Debye series are obtained as follows. Consider a spherically incoming TE partial wave $\xi_{l}^{(2)}\left(n_{2} k r\right)$ in region 2 incident on the sphere surface. Writing the scalar radiation potentials in regions 1 and 2 as

$$
\begin{aligned}
\Psi_{1, l}(r, \theta, \phi)= & \sum_{l=1}^{\infty} n_{1}\left\{\left(i^{l}\right)(2 l+1) /[l(l+1)]\right\} \\
& \times T_{21, l} \xi_{l}^{(2)}\left(n_{1} k r\right) P_{l}^{1}[\cos (\theta)] \sin (\phi), \\
\Psi_{2, l}(r, \theta, \phi)= & \sum_{l=1}^{\infty} n_{2}\left\{\left(i^{l}\right)(2 l+1) /[l(l+1)]\right\}\left[\xi_{l}^{(2)}\left(n_{2} k r\right)\right. \\
& \left.+R_{212, l} \xi_{l}^{(1)}\left(n_{2} k r\right)\right] P_{l}^{1}[\cos (\theta)] \sin (\phi)
\end{aligned}
$$

and matching the boundary conditions for the electric and magnetic fields at the sphere surface, one obtains

$$
T_{21, l}=-2 i n_{2} /\left[\left(N_{12, l}+Q_{12, l}\right)+i\left(D_{12, l}-P_{12, l}\right)\right]
$$

as the transmission amplitude of the partial wave $l$ from region 2 to region 1 and

$$
\begin{aligned}
R_{212, l}= & {\left[-\left(N_{12, l}-Q_{12, l}\right)+i\left(D_{12, l}+P_{12, l}\right)\right] /\left[\left(N_{12, l}\right.\right.} \\
& \left.\left.+Q_{12, l}\right)+i\left(D_{12, l}-P_{12, l}\right)\right]
\end{aligned}
$$

as the amplitude of the partial wave in region 2 reflecting off region 1 back into region 2 . The refractive indices $n_{1}$ and $n_{2}$ explicitly appear in the scalar radiation potentials of Eqs. (16a)-(16b) in order to make the results for the reflection and transmission amplitudes as symmetric as possible. This was not done in Refs. 21,22 , and 24 , leading to various refractiveindex ratios that do not appear here. If one had a TE spherically outgoing partial-wave $\xi_{l}^{(1)}\left(n_{1} k r\right)$ in region 1 incident on the sphere surface, the scalar radiation potentials would be

$$
\begin{aligned}
\Psi_{1, l}(r, \theta, \phi)= & \sum_{l=1}^{\infty} n_{1}\left\{\left(i^{l}\right)(2 l+1) /[l(l+1)]\right\}\left[\xi_{l}^{(1)}\left(n_{1} k r\right)\right. \\
& \left.+R_{121, l} \xi_{l}^{(2)}\left(n_{1} k r\right)\right] P_{l}^{1}[\cos (\theta)] \sin (\phi),
\end{aligned}
$$

$$
\begin{aligned}
\Psi_{2, l}(r, \theta, \phi)= & \sum_{l=1}^{\infty} n_{2}\left\{\left(i^{l}\right)(2 l+1) /[l(l+1)]\right\} \\
& \times T_{12, l} \xi_{l}{ }^{(1)}\left(n_{2} k r\right) P_{l}^{1}[\cos (\theta)] \sin (\phi) .
\end{aligned}
$$

Matching the boundary conditions for the fields at the surface of the sphere, one obtains 


$$
T_{12, l}=-2 i n_{1} /\left[\left(N_{12, l}+Q_{12, l}\right)+i\left(D_{12, l}-P_{12, l}\right)\right]
$$

as the transmission amplitude of the partial wave $l$ from region 1 to region 2 and

$$
\begin{aligned}
R_{121, l}= & {\left[-\left(N_{12, l}-Q_{12, l}\right)-i\left(D_{12, l}+P_{12, l}\right)\right] /\left[\left(N_{12, l}\right.\right.} \\
& \left.\left.+Q_{12, l}\right)+i\left(D_{12, l}-P_{12, l}\right)\right]
\end{aligned}
$$

as the amplitude of the partial wave in region 1 reflecting off region 2 back into region 1 . The formulas for the TM partial-wave transmission and reflection amplitudes are identical. Finally, with the help of Eq. (6), the Debye series decomposition of the partialwave scattering and interior amplitudes is

$$
a_{12, l}, b_{12, l}=(1 / 2)\left[1-R_{212, l}-T_{21, l} T_{12, l} /\left(1-R_{121, l}\right)\right],
$$

$$
c_{12, l}, d_{12, l}=T_{21, l} /\left(1-R_{121, l}\right) .
$$

The infinite series of multiple internal reflections of the partial wave is generated by a Taylor series expanding the denominator of Eqs. (22a)-(22b). It should be noted that the scalar radiation potentials in Eqs. (16a), (16b), (19a), and (19b) contain only spherically incoming and outgoing waves. Replacing some of these by the spherical standing wave $\psi_{l}(n k r)$ generates a different expansion of the partial-wave scattering amplitudes that has a different physical interpretation. This will be discussed more fully in Section 4.

\section{B. Coated Sphere and Multilayer Sphere}

The pattern of the Debye series for scattering by a homogeneous sphere is found, after much algebra, to be repeated in exactly the same way for scattering by a layered sphere with $M \geq 2$. For a coated sphere, i.e., $M=2$, we already combined interfaces 12 and 23 with Eqs. (9a)-(9b) into a single scattering unit, here called $\gamma$. Writing the scalar radiation potentials $\Psi_{1}(r, \theta, \phi)$ in the core, $\Psi_{2}(r, \theta, \phi)$ in the coating, and $\Psi_{3}(r, \theta, \phi)$ in the exterior medium as in Eqs. (16a), (16b), (19a), and (19b) and matching boundary conditions of the electric and magnetic fields, we obtain

$$
\begin{aligned}
& T_{31}=2 i n_{2} n_{3} /\left[\left(N_{123}+Q_{123}\right)+i\left(D_{123}-P_{123}\right)\right], \\
& T_{13}=2 i n_{1} n_{2} /\left[\left(N_{123}+Q_{123}\right)+i\left(D_{123}-P_{123}\right)\right],
\end{aligned}
$$

as the TE and TM amplitudes of a partial wave in region 3 reflecting back and forth between the two interfaces any number of times and finally ending up in region 1 and of a partial wave beginning in region 1 and ending up in region 3 , respectively. Similarly,

$$
\begin{aligned}
R_{3 \gamma 3}= & {\left[-\left(N_{123}-Q_{123}\right)+i\left(D_{123}+P_{123}\right)\right] /\left[\left(N_{123}+Q_{123}\right)\right.} \\
& \left.+i\left(D_{123}-P_{123}\right)\right], \\
R_{1 \gamma 1}= & {\left[-\left(N_{123}-Q_{123}\right)-i\left(D_{123}+P_{123}\right)\right] /\left[\left(N_{123}+Q_{123}\right)\right.} \\
& \left.+i\left(D_{123}-P_{123}\right)\right]
\end{aligned}
$$

are the amplitudes of a TE or TM partial wave beginning in region 3 , successively reflecting back and forth between the two interfaces any number of times, and ending up back in region 3 , and of a partial wave beginning in region 1 , reflecting off the interfaces, and ending up back in region 1, respectively. From Eq. (10), the partial-wave scattering and core amplitudes are found to be

$$
\begin{aligned}
& a_{123}, b_{123}=(1 / 2)\left[1-R_{3 \gamma 3}-T_{31} T_{13} /\left(1-R_{1 \gamma 1}\right)\right], \\
& c_{123}, d_{123}=T_{31} /\left(1-R_{1 \gamma 1}\right) .
\end{aligned}
$$

The composite interface transmission and reflection amplitudes may be written in terms of the singleinterface transmission and reflection amplitudes as

$$
\begin{aligned}
T_{31} & =T_{32} T_{21} /\left(1-R_{212} R_{232}\right), \\
T_{13} & =T_{12} T_{23} /\left(1-R_{212} R_{232}\right), \\
R_{3 \gamma 3} & =R_{323}+T_{32} R_{212} T_{23} /\left(1-R_{212} R_{232}\right), \\
R_{1 \gamma 1} & =R_{121}+T_{12} R_{232} T_{21} /\left(1-R_{212} R_{232}\right),
\end{aligned}
$$

and, substituting Eqs. (28)-(31) into Eqs. (27a)-(27b), the coated sphere partial-wave scattering amplitudes can be written in terms of the single-interface transmission and reflection amplitudes. The results are identical to Eqs. (37), (38), (42), and (43) of Ref. 22. It should be stressed that the composite amplitudes $T_{31}$, $T_{13}, R_{3 \gamma 3}$, and $R_{1 \gamma 1}$ are multiple scattering amplitudes, containing the infinite series of all the interactions of the partial wave with all the interfaces between the starting and ending regions.

The same pattern continues for an $M$-layer sphere. Again using incoming and outgoing spherical waves everywhere in the scalar radiation potentials $\Psi_{1}(r, \theta, \phi)$ through $\Psi_{M+1}(r, \theta, \phi)$, we find that

$$
\begin{aligned}
T_{M+1,1}= & 2 i n_{2} n_{3} \ldots n_{M+1} /\left[\left(N_{12 \ldots M+1}+Q_{12 \ldots M+1}\right)\right. \\
& \left.+i\left(D_{12 \ldots M+1}-P_{12 \ldots M+1}\right)\right] \\
T_{1, M+1}= & 2 i n_{1} n_{2} \ldots n_{M} /\left[\left(N_{12 \ldots M+1}+Q_{12 \ldots M+1}\right)\right. \\
& \left.+i\left(D_{12 \ldots M+1}-P_{12 \ldots M+1}\right)\right]
\end{aligned}
$$

are the partial-wave transmission amplitudes from the exterior medium multiply scattering between all the layers of the sphere, which we here call $\Gamma$, to the core, and those from the core multiply scattering between all the layers to the exterior medium, respectively. Similarly, 


$$
\begin{aligned}
R_{M+1, \Gamma, M+1} & =\frac{-\left(N_{12 \ldots M+1}-Q_{12 \ldots M+1}\right)+i\left(D_{12 \ldots M+1}+P_{12 \ldots M+1}\right)}{\left(N_{12 \ldots M+1}+Q_{12 \ldots M+1}\right)+i\left(D_{12 \ldots M+1}-P_{12 \ldots M+1}\right)}, \\
R_{1, \Gamma, 1} & =\frac{-\left(N_{12 \ldots M+1}-Q_{12 \ldots M+1}\right)-i\left(D_{12 \ldots M+1}+P_{12 \ldots M+1}\right)}{\left(N_{12 \ldots M+1}+Q_{12 \ldots M+1}\right)+i\left(D_{12 \ldots M+1}-P_{12 \ldots M+1}\right)}
\end{aligned}
$$

are the amplitudes of the partial wave starting in the exterior region, multiply scattering between all the layers, and ending up back in the exterior medium, and those starting in the core, multiply scattering between all of the layers, and ending up back in the core, respectively. The $M$-layer sphere partial-wave scattering amplitudes again assume the form

$$
\begin{aligned}
a_{12 \ldots M+1}, b_{12 \ldots M+1}= & (1 / 2)\left[1-R_{M+1, \Gamma, M+1}\right. \\
& -T_{M+1,1} T_{1, M+1} /\left(1-R_{1, \Gamma, 1}\right] .
\end{aligned}
$$

The multiple-scattering structure of the overall transmission and reflection amplitudes of Eqs. (32)-(35) is made apparent by writing them in terms of lower-order transmission and reflection amplitudes. For example, for $M=4$ we can combine the 12 and 23 interfaces into the unit $\alpha$ and the 34 and 45 interfaces into the unit $\beta$. We then obtain

$$
\begin{aligned}
T_{51} & =T_{53} T_{31} /\left(1-R_{3 \alpha 3} R_{3 \beta 3}\right), \\
T_{15} & =T_{13} T_{35} /\left(1-R_{3 \alpha 3} R_{3 \beta 3}\right), \\
R_{5 \Gamma 5} & =R_{5 \beta 5}+T_{53} R_{3 \alpha 3} T_{35} /\left(1-R_{3 \alpha 3} R_{3 \beta 3}\right), \\
R_{1 \Gamma 1} & =R_{1 \alpha 1}+T_{13} R_{3 \beta 3} T_{31} /\left(1-R_{3 \alpha 3} R_{3 \beta 3}\right),
\end{aligned}
$$

and substitution of Eqs. (28)-(31) into Eqs. (37)-(40) gives the composite transmission and reflection amplitudes in terms of single-interface transmission and reflection amplitudes.

\section{Order of Scattering Expansion}

Chew ${ }^{14}$ and Fuller ${ }^{15,16}$ have derived an alternate expansion of the multilayer sphere partial-wave scattering amplitudes. For the $M=1$ homogeneous sphere problem, in addition to Eqs. (7a) and (7b) they considered a spherically outgoing wave in region 1 partially transmitted and reflected at the sphere surface. But, instead of Eqs. (19a) and (19b), they used a spherical standing wave $\psi_{l}\left(n_{1} k r\right)$ for the reflected wave, giving the TE scalar radiation potentials

$$
\begin{aligned}
\Psi_{1, l}(r, \theta, \phi)= & \sum_{l=1}^{\infty} n_{1}\left\{\left(i^{l}\right)(2 l+1) /[l(l+1)]\right\}\left[\xi_{l}^{(1)}\left(n_{1} k r\right)\right. \\
& \left.+R_{121, l^{\prime}} \psi_{l}\left(n_{1} k r\right)\right] P_{l}^{1}[\cos (\theta)] \sin (\phi), \\
\Psi_{2, l}(r, \theta, \phi)= & \sum_{l=1}^{\infty} n_{2}\left\{\left(i^{l}\right)(2 l+1) /[l(l+1)]\right\} \\
& \times T_{12, l^{\prime}} \xi_{l}^{(1)}\left(n_{2} k r\right) P_{l}^{1}[\cos (\theta)] \sin (\phi) .
\end{aligned}
$$

Matching the boundary conditions for the electric and magnetic fields at the sphere surface, they obtained

$$
\begin{aligned}
T_{12, l^{\prime}}= & -i n_{1} /\left(N_{12, l}+i \quad D_{12, l}\right) \\
R_{121, l^{\prime}}= & {\left[-\left(N_{12, l}-Q_{12, l}\right)-i\left(D_{12, l}+P_{12, l}\right)\right] /\left(N_{12, l}\right.} \\
& \left.+i D_{12, l}\right)
\end{aligned}
$$

in the notation used here. The benefit of this approach is that if it is applied to the $M$-layer sphere problem, one has the symmetric situation of a spherically outgoing wave and a spherical standing wave in every region. For the $M=2$ coated sphere problem they obtained

$$
\begin{aligned}
& a_{123}=a_{23}+a_{12} c_{23} T_{23}{ }^{\prime} /\left(1+a_{12} R_{232}{ }^{\prime}\right), \\
& b_{123}=b_{23}+b_{12} d_{23} T_{23}{ }^{\prime} /\left(1+b_{12} R_{232}{ }^{\prime}\right)
\end{aligned}
$$

for the coated sphere partial-wave scattering amplitudes. The way in which this result differs from the Debye series of Eq. (27a) is as follows. Whereas a Taylor series expansion of the denominator of the Debye series decomposes the partial-wave scattering amplitudes into a sum of terms containing the fundamental interactions of diffraction, exterior reflection, and transmission following any number of internal reflections, a Taylor series expansion of the denominator of Eqs. (44a)-(44b) decomposes the partial-wave scattering amplitudes in terms of powers of the homogeneous sphere scattering amplitudes $a_{12}, b_{12}, a_{23}$, and $b_{23}$. This is called the order of scattering expansion and was extended by these authors to an $M$-layer sphere via the progressive iteration method described above. 


\section{Amplification of External Reflection by a Spherical Bragg Grating}

In order to test the multilayer sphere Debye series formulas of Section 3, the scattered intensity as a function of the scattering angle $\theta$ was numerically computed for an $M=64$ layer sphere by using the parallel iteration method described in Subsection 2.D. The radius and refractive index of the core particle were chosen to be $A_{1}=20.0 \mu \mathrm{m}$ and $n_{1}=1.50$. Layered onto this core are 63 concentric shells, each of thickness $\Delta=0.1 \mu \mathrm{m}$, with the refractive index alternating between 1.55 and 1.50 , i.e., $A_{2}=$ $20.1 \mu \mathrm{m}$ and $n_{2}=1.55, A_{3}=20.2 \mu \mathrm{m}$ and $n_{3}=$ $1.50, A_{4}=20.3 \mu \mathrm{m}$ and $n_{4}=1.55$ all the way out to $A_{64}=26.3 \mu \mathrm{m}$ and $n_{64}=1.55$. The external medium was taken to be air with $n_{65}=1.0$. These concentric layers form a spherical Bragg grating covering the core particle. If one had a flat planar Bragg grating consisting of a large number of sheets $\Delta=0.1 \mu \mathrm{m}$ thick whose refractive index alternated between 1.50 and 1.55 and a plane wave in air were incident on the grating with the angle of incidence $\theta_{i}$, strong constructive interference for reflection would occur when

$$
\lambda_{r}=4 n_{\mathrm{ave}} \Delta \cos \left(\theta_{t}\right)
$$

where

$$
\sin \left(\theta_{i}\right)=n_{\text {ave }} \sin \left(\theta_{t}\right)
$$

and $n_{\text {ave }}$ is the average refractive index of the grating. At normal incidence Eqs. (45) and (46) give $\lambda_{r}$ $=0.61 \mu \mathrm{m}$ for $n_{\text {ave }}=1.525$.

As a baseline for what to expect for scattering by the $M=64$ spherical Bragg grating, Fig. 1 shows the scattered intensity for an unpolarized plane wave with $\lambda=0.575 \mu \mathrm{m}$ incident on a homogeneous sphere of refractive index $n_{1}=1.50$ and radius $A_{1}=$ $26.3 \mu \mathrm{m}$. Also shown is the intensity for diffraction plus the Debye series external reflection term of Eq. (18). (Diffraction and external reflection must be combined in order to guarantee that the Mie sum over

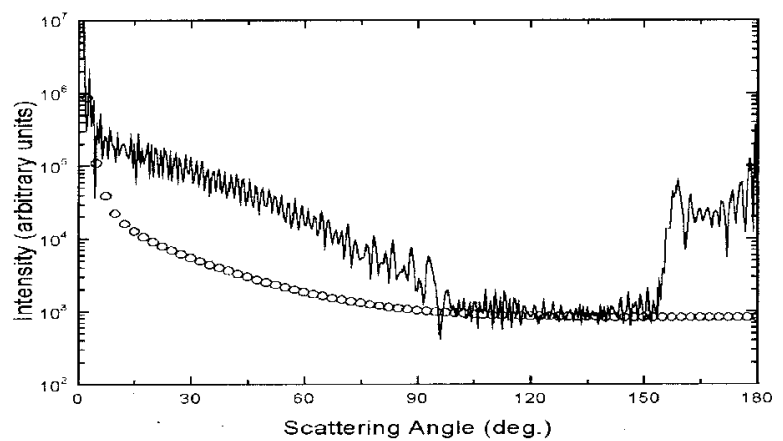

Fig. 1. Scattered intensity as a function of the scattering angle $\theta$ for an unpolarized plane wave in air with $\lambda=0.575 \mu \mathrm{m}$ incident on a homogeneous sphere of radius $26.3 \mu \mathrm{m}$ and refractive index 1.50. The solid line is the scattered intensity and the open circles are the diffraction-plus-external reflection intensity. partial waves converges. ${ }^{19}$ ) In Fig. 1 the angular region $10^{\circ} \leq \theta \leq 95^{\circ}$ is dominated by interference between transmission and the much weaker external reflection. The first-order rainbow appears prominently at $\theta=158^{\circ}$, and the second-order rainbow at $\theta=91^{\circ}$ is in part obscured by transmission, which in ray theory is extinguished for $\theta \geq 96^{\circ}$. The only angular region dominated by external reflection is $95^{\circ}$ $\leq \theta \leq 155^{\circ}$ in Alexander's dark band ${ }^{33}$ between the first- and second-order rainbows. In ray theory the unpolarized external reflection intensity is

$$
L_{\text {ref }}(\theta)=\left(\pi A_{1} / \lambda\right)^{2}\left(R_{212}^{\text {ray, } \mathrm{TE}}+R_{212}^{\text {ray, } \mathrm{TM}}\right) / 2,
$$

with

$$
\begin{aligned}
R_{212}{ }^{\text {ray, TE }}= & {\left[n_{2} \cos \left(\theta_{i}\right)-n_{1} \cos \left(\theta_{t}\right)\right]^{2} /\left[n_{2} \cos \left(\theta_{i}\right)\right.} \\
& \left.+n_{1} \cos \left(\theta_{t}\right)\right]^{2}, \\
R_{212}{ }^{\text {ray, TM }}= & {\left[n_{1} \cos \left(\theta_{i}\right)-n_{2} \cos \left(\theta_{t}\right)\right]^{2} /\left[n_{1} \cos \left(\theta_{i}\right)\right.} \\
& \left.+n_{2} \cos \left(\theta_{t}\right)\right]^{2}, \\
n_{2} \sin \left(\theta_{i}\right)= & n_{1} \sin \left(\theta_{t}\right), \\
\theta= & \pi-2 \theta_{i} .
\end{aligned}
$$

For the situation of Fig. 1 the ray theory external reflection intensity decreases monotonically by a factor of 25 from $2.06 \times 10^{4}$ at $\theta=0^{\circ}$ to 826 at $\theta$ $=180^{\circ}$. This behavior is apparent in the Debye series plot in Fig. 1.

Figure 2 shows the homogeneous sphere unpolarized Mie intensity of Fig. 1 along with the scattered intensity for the $M=64$ layer sphere for an incident plane wave with $\lambda=0.575 \mu \mathrm{m}$. The greatest differences between the two intensities are (i) a narrow decrease by about a factor of 15 at $\theta \approx 20^{\circ}$, (ii) a broad amplification by about a factor of 20 in Alexander's dark band for $110^{\circ} \leq \theta \leq 135^{\circ}$, and (iii) a smaller

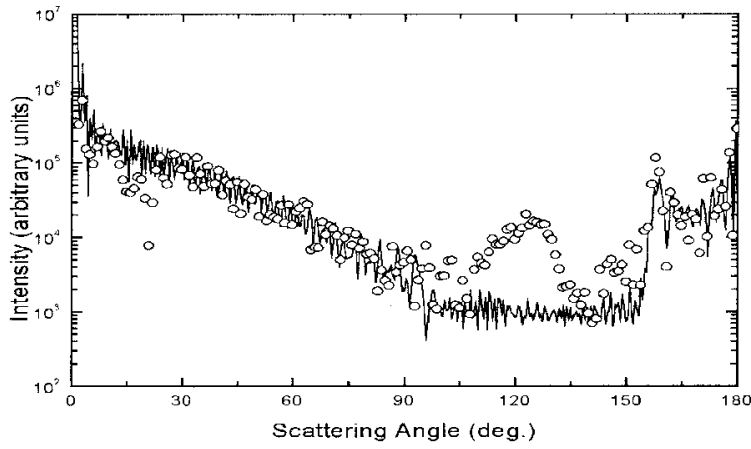

Fig. 2. Scattered intensity as a function of the scattering angle $\theta$ for an unpolarized plane wave in air with $\lambda=0.575 \mu \mathrm{m}$ incident on the homogeneous sphere of Fig. 1 (solid curve) and on a 63-layer grating covering a core particle of radius $20.0 \mu \mathrm{m}$ and refractive index 1.50 (open circles). Each grating layer is $0.1 \mu \mathrm{m}$ thick, and the refractive index in successive layers alternates between 1.55 and 1.50 . 


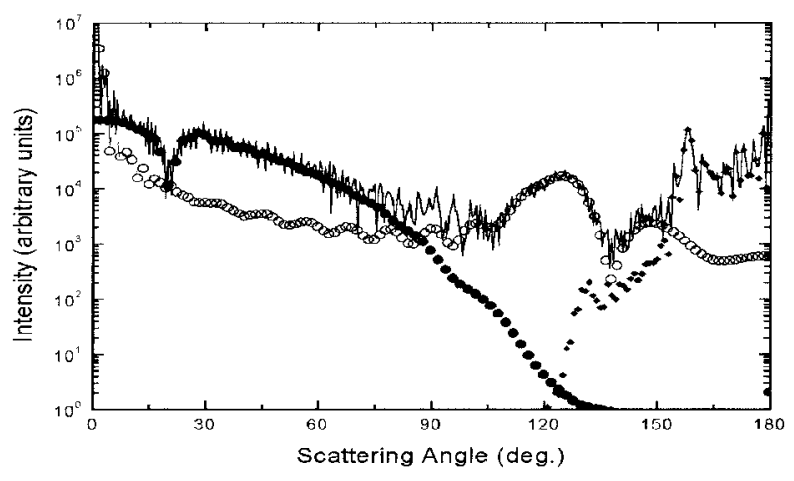

Fig. 3. Scattered intensity as a function of the scattering angle $\theta$ for an unpolarized plane wave in air with $\lambda=0.575 \mu \mathrm{m}$ incident on the 63-layer grating covering the core particle of Fig. 2. Solid curve, scattered intensity; open circles, diffraction-plus-external reflection intensity; filled circles, transmitted intensity; filled diamonds, intensity for transmission following one internal multiple reflection between the layers.

amplification for $145^{\circ} \leq \theta \leq 155^{\circ}$. In Fig. 3 the $M$ $=64$ scattered intensity is reproduced along with the Debye series terms of Eqs. (32)-(36) for diffractionplus-external reflection from the external medium back and forth between all the layers into the external medium again, transmission, and transmission following a single internal reflection from the core back and forth between all the layers and back into the core again. Whereas the external reflection term for the homogeneous sphere in Fig. 1 was devoid of interesting structure, the corresponding term for the $M=64$ layer sphere has much interesting structure since it is essentially the spherical Bragg grating reflectivity angular spectrum. According to Eqs. (45), (46), and (50), for $\lambda_{r}=0.575 \mu \mathrm{m}$, maximum constructive interference for external reflection from the Bragg grating layers should occur at $\theta \approx 118.8^{\circ}$ for $n_{\text {ave }}=1.525$ and is the source of the broad amplification observed in Fig. 2 for $110^{\circ} \leq \theta \leq 135^{\circ}$. Similarly, the small amplification for $145^{\circ} \leq \theta \leq 155^{\circ}$ is due to the first angular sidelobe of the spherical Bragg grating reflectivity spectrum. Since external reflection is more than $90 \%$ of the geometrical limit in the large broad amplification region, only a small amount of power is transmitted through the layers to the core. In ray theory this dip in transmission should occur at $\theta \approx 22.2^{\circ}$ and is the source of the deep dip in the transmitted intensity observed in Fig. 2 at $\theta$ $\approx 20^{\circ}$. As was found in Ref. 22 , the first-order rainbow for a coated sphere breaks into two components. The stronger $\beta$ component in Fig. 3 at $\theta \approx 158^{\circ}$ corresponds to the one internal reflection occurring at the surface of the multilayer sphere, and the weaker $a$ component in Fig. 3 at $\theta \approx 132^{\circ}$ corresponds to the one internal reflection occurring at the various interior interfaces. For a given wavelength, the fact that Eqs. (45) and (46) predict that the reflection peak occurs at a single $\theta_{i}$ is equivalent ${ }^{34}$ to the fact that

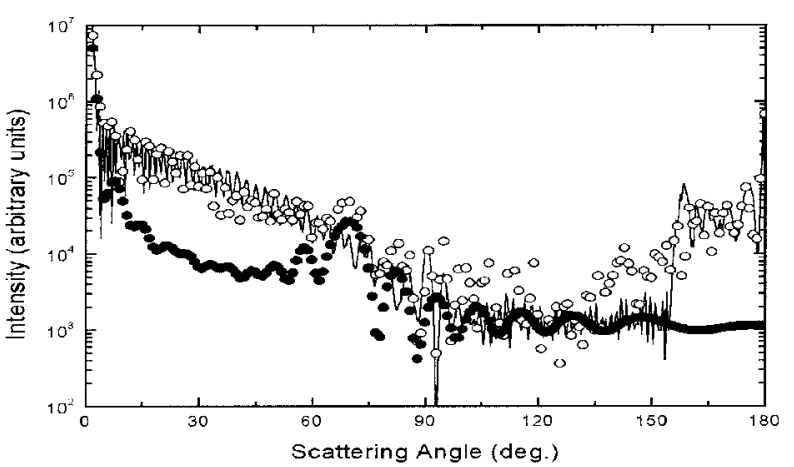

(a)

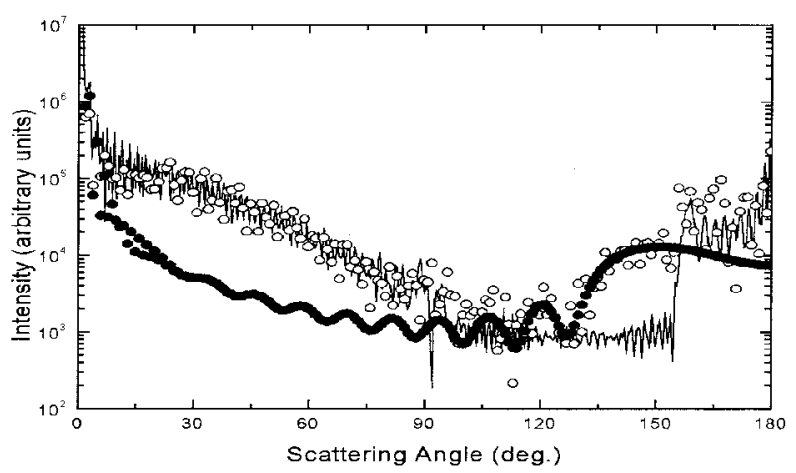

(c)

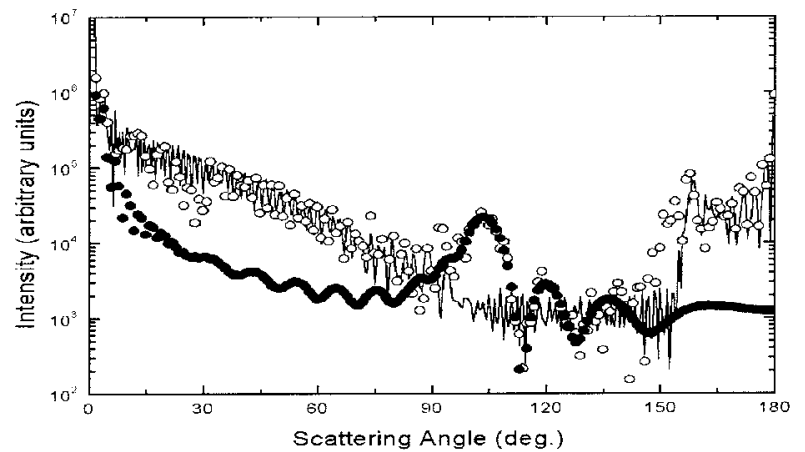

(b)

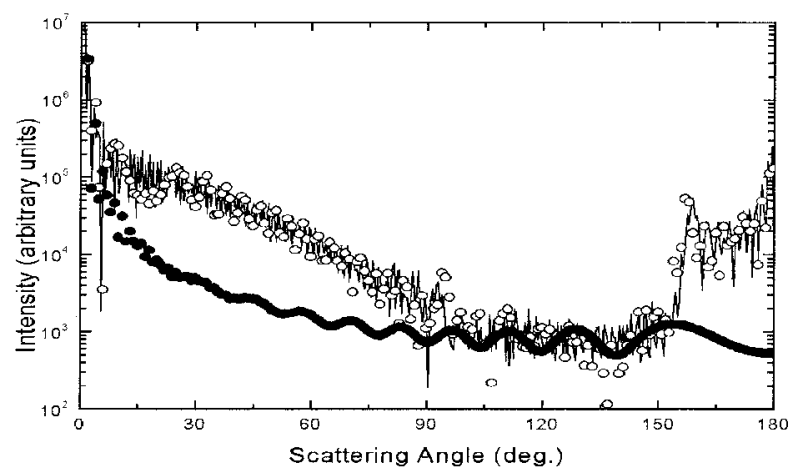

(d)

Fig. 4. Scattered intensity as a function of the scattering angle $\theta$ for an unpolarized plane wave in air with (a) $\lambda=0.50 \mu \mathrm{m}$, (b) $\lambda$ $=0.55 \mu \mathrm{m}$, (c) $\lambda=0.60 \mu \mathrm{m}$, and (d) $\lambda=0.65 \mu \mathrm{m}$ incident on the homogeneous sphere of Fig. 1 (solid curve) and the 63-layer grating covering the core particle of Fig. 2 (open circles). The filled circles are the multilayer sphere diffraction-plus-external reflection intensity. 
only some of the partial waves in the Mie sum are at a reflection resonance while most are not. ${ }^{16}$

Figures 4(a), 4(b), 4(c), and 4(d) show the scattered intensity for the same $M=1$ homogeneous sphere, the same $M=64$ layer sphere, and the layered sphere diffraction-plus-external reflection intensity for $\lambda=0.50,0.55,0.60,0.65 \mu \mathrm{m}$. According to Eqs. (45), (46), and (50), for $n_{\text {ave }}=1.525$, the external reflection peak should occur at $\theta=58.3^{\circ}$ for $\lambda_{r}$ $=0.50 \mu \mathrm{m}, \theta=97.5^{\circ}$ for $\lambda_{r}=0.55 \mu \mathrm{m}$, and $\theta$ $=148.1^{\circ}$ for $\lambda_{r}=0.60 \mu \mathrm{m}$. For $\lambda=0.65 \mu \mathrm{m}$, no external reflection maxima are predicted. The observed angle of the reflection peak in Figs. 4(a)-4(c) is slightly larger than the flat interface prediction, the difference decreasing as the reflection peak is closer to backscattering, corresponding to normal incidence. This difference is most likely due to the curvature of the interfaces. As $\theta_{i}$ increases, the incident rays experience differing amounts of curvature of the different layers, leading to many values of $\theta_{t}$ rather than the single $\theta_{t}$ obtained for incidence on a flat stack of interfaces. For $\lambda$ of 0.50 and $0.55 \mu \mathrm{m}$ the peak reflectivity is again more than $90 \%$ of the geometrical limit, but for $\lambda=0.50 \mu \mathrm{m}$ the reflectivity peak near $70^{\circ}$ is largely lost in the transmitted light. Only when the external reflection peak enters Alexander's dark band for $\lambda=0.55 \mu \mathrm{m}$ does it dominate the scattered intensity. For $\lambda=0.60 \mu \mathrm{m}$, the 63 layers covering the core particle act as a Fabry-Perot resonator at $\theta$ $\approx 180^{\circ}$, raising the external reflection intensity there by an order of magnitude. By $\lambda=0.65 \mu \mathrm{m}$, the light multiply reflected by the 63 layers is no longer able to constructively interfere at any scattering angle. As a result, the $M=64$ intensity closely resembles that of the homogeneous sphere, and the $M=64$ diffractionplus-external reflection intensity is starting to settle down and resemble that of the homogeneous sphere in Fig. 1. It should also be noted that the $\beta$ first-order rainbow angle decreases from $\theta \approx 158^{\circ}$ for $\lambda$ $=0.575 \mu \mathrm{m}$ to $\theta \approx 156^{\circ}$ for $\lambda=0.55 \mu \mathrm{m}$ to $\theta$ $\approx 145^{\circ}$ for $\lambda=0.50 \mu \mathrm{m}$, while the $\alpha$ first-order rainbow remains almost 3 orders of magnitude weaker than the $\beta$ rainbow at $\theta \approx 132^{\circ}$.

If the number of spherical Bragg grating layers were larger, the angular width of the external reflection peak would narrow but would be limited in height by the geometrical $100 \%$ reflectivity constraint. Thus the reflectivity peak of such a multilayered sphere would strongly make its presence known in the scattered intensity only for incident wavelengths less than $\lambda_{r}$ of Eq. (45) for normal incidence and only when the intensity peak occurs between the first- and second-order rainbows. If white light were to illuminate such a multilayer sphere with $\Delta$ $=0.1 \mu \mathrm{m}$, Alexander's dark band would glow from reddish near the first-order rainbow to greenish near the second-order rainbow, giving the visual appearance of an external reflection rainbow.

In summary, a large variety of radial inhomogeneities can be accurately modeled by using a radially stratified sphere. Both the partial-wave scattering amplitudes and the Debye series transmission and reflection amplitudes are combinations of the four basic TE or TM amplitudes and can be computed by using either the progressive or parallel iteration methods. These combined amplitudes correspond to combining a number of adjacient interfaces into a single scattering unit, with each combined amplitude containing the multiple scattering series of interactions of the partial wave with all the interfaces that comprise the scattering unit. The specific example chosen for numerical study here illustrates that Debye series methods are of great utility in interpreting novel structures appearing in the scattered intensity.

This research was supported in part by the National Aeronautics and Space Administration under cooperative agreement NCC3-909.

\section{References}

1. K. A. Fuller and D. W. Mackowski, "Electromagnetic scattering by compounded spherical particles," in Light Scattering by Nonspherical Particles, M. I. Mishchenko, J. W. Hovenier, and L. D. Travis, eds. (Academic, 2000), pp. 225-272.

2. P. Massoli, "Rainbow refractometry applied to radially inhomogeneous spheres: the critical case of evaporating droplets," Appl. Opt. 37, 3227-3235 (1998).

3. A. L. Aden and M. Kerker, "Scattering of electromagnetic waves from two concentric spheres," J. Appl. Phys. 22, 12421246 (1951).

4. M. Kerker, J. P. Kratohvil, and E. Matijevic, "Light scattering functions for concentric spheres. Total scattering coefficients, $m_{1}=2.1050, m_{2}=1.4821$," J. Opt. Soc. Am. 52, 551-561 (1962).

5. R. Bhandari, "Scattering coefficients for a multilayered sphere: analytic expressions and algorithms," Appl. Opt. 24, 19601967 (1985).

6. M. Born and E. Wolf, Principles of Optics, 6th ed. (Cambridge U. Press, 1980), pp. 51-70.

7. W. C. Chew, Waves and Fields in Inhomogeneous Media (Van Nostrand Reinhold, 1990), pp. 45-53.

8. O. B. Toon and T. P. Ackerman, "Algorithms for the calculation of scattering by stratified spheres," Appl. Opt. 20, 3657-3660 (1981).

9. D. W. Mackowski, R. A. Altenkirch, and M. P. Menguc, "Internal absorption cross sections in a stratified sphere," Appl. Opt. 29, 1551-1559 (1990).

10. Z. S. Wu and Y. P. Wang, "Electromagnetic scattering for multilayered sphere: recursive algorithms," Radio Sci. 26, 1393-1401 (1991).

11. L. Kai and P. Massoli, "Scattering of electromagnetic-plane waves by radially inhomogeneous spheres: a finely stratified sphere model," Appl. Opt. 33, 501-511 (1994).

12. B. R. Johnson, "Light scattering by a multilayer sphere," Appl. Opt. 35, 3286-3296 (1996).

13. Z. S. Wu, L. X. Guo, K. F. Ren, G. Gouesbet, and G. Grehan, "Improved algorithm for electromagnetic scattering of plane waves and shaped beams by multilayered spheres," Appl. Opt. 36, 5188-5198 (1997).

14. Ref. 7, pp. 187-193.

15. K. A. Fuller, "Scattering of light by coated spheres," Opt. Lett. 18, 257-259 (1993).

16. D. D. Smith and K. A. Fuller, "Photonic bandgaps in Mie scattering by concentric stratified spheres," J. Opt. Soc. Am. B 19, 2449-2455 (2002).

17. P. Debye, "Das elektromagnetische Feld um einen Zylinder und die Theorie des Regenbogens," Phys. Z. 9, 775-778 (1908) [reprinted and translated into English in P. L. Marston, ed., 
Selected Papers on Geometrical Aspects of Scattering, Vol. 89 of SPIE Milestone Series, SPIE 1994), pp. 198-204].

18. B. van der Pol and H. Bremmer, "Diffraction of electromagnetic waves from an electrical point source round a finitely conducting sphere with applications to radiotelegraphy and the theory of the rainbow," Philos. Mag. 24, 141-176, 825-864 (1937).

19. H. M. Nussenzveig, "High-frequency scattering by a transparent sphere. I. Direct reflection and transmission," J. Math. Phys. 10, 82-124 (1969).

20. H. M. Nussenzveig, "High-frequency scattering by a transparent sphere. II. Theory of the rainbow and the glory," J. Math. Phys. 10, 125-176 (1969).

21. J. A. Lock, "Cooperative effects among partial waves in Mie scattering," J. Opt. Soc. Am. A 5, 2032-2044 (1988).

22. J. A. Lock, J. M. Jamison, and C.-Y. Lin, "Rainbow scattering by a coated sphere," Appl. Opt. 33, 4677-4690, 4960 (1994).

23. J. A. Lock and C. L. Adler, "Debye-series analysis of the firstorder rainbow produced in scattering of a diagonally incident plane wave by a circular cylinder," J. Opt. Soc. Am. A 14, 1316-1328 (1997).

24. E. A. Hovenac and J. A. Lock, "Assessing the contributions of surface waves and complex rays to far-field Mie scattering by use of the Debye series," J. Opt. Soc. Am. A 9, 781-795 (1992).

25. P. Yeh, A. Yariv, and C.-S. Hong, "Electromagnetic propagation in periodic stratified media. I. General theory," J. Opt. Soc. Am. 67, 423-438 (1977).

26. A. E. Siegman, "Bragg diffraction of a Gaussian beam by a crossed-Gaussian volume grating," J. Opt. Soc. Am. 67, 545550 (1977).

27. M. Abramowitz and I. A. Stegun, eds., Handbook of Mathematical Functions (National Bureau of Standards, 1964), Sec. 10.3, p. 445.

28. H. C. van de Hulst, Light Scattering by Small Particles (Dover, 1981), p. 125.

29. M. Kerker, The Scattering of Light and Other Electromagnetic Radiation (Academic, 1969), p. 47.

30. C. F. Bohren and D. R. Huffman, Absorption and Scattering of Light by Small Particles (Wiley-Interscience, 1983), pp. 111113.

31. Ref. 29 , pp. $189-195$.

32. Ref. 30, pp. 181-183, 483-489.

33. H. M. Nussenzveig, "The theory of the rainbow," Sci. Am. 236(4), 116-127 (1977).

34. Ref. 28 , pp. 208-209. 